\title{
Systematic Literature Review of DPP-4 Inhibitors in Patients with Type 2 Diabetes Mellitus and Renal Impairment
}

\author{
Merlin C. Thomas (D) Päivi M. Paldánius · Rajeev Ayyagari · \\ Siew Hwa Ong · Per-Henrik Groop
}

Received: May 18, 2016/Published online: August 8, 2016

(C) The Author(s) 2016. This article is published with open access at Springerlink.com

\begin{abstract}
Introduction: Dipeptidyl peptidase-4 (DPP-4) inhibitors are widely used in the management of patients with type 2 diabetes mellitus (T2DM)

Enhanced content To view enhanced content for this article go to http://www.medengine.com/Redeem/ 19E4F06066D88D9E.

Electronic supplementary material The online version of this article (doi:10.1007/s13300-016-0189-4) contains supplementary material, which is available to authorized users.
\end{abstract}

M. C. Thomas $(\bowtie)$ P.-H. Groop

Baker IDI Heart and Diabetes Institute, Melbourne, Australia

e-mail: merlin.thomas@bakeridi.edu.au

P. M. Paldánius · S. H. Ong

Novartis Pharma AG, Basel, Switzerland

R. Ayyagari

Analysis Group Inc., Boston, MA, USA

Present Address:

S. H. Ong

Vifor Pharma Ltd., Glattbrugg, Switzerland

P.-H. Groop

Abdominal Centre Helsinki, University of Helsinki and Helsinki University Hospital, Helsinki, Finland

P.-H. Groop

Folkhälsan Institute of Genetics, Folkhälsan

Research Centre, Biomedicum Helsinki, Helsinki, Finland and renal impairment (RI). A systematic literature review was performed to compare the efficacy and safety of DPP-4 inhibitors in patients with T2DM and RI.

Methods: We searched EMBASE, MEDLINE, and the Cochrane Central Register of Controlled Trials (cut-off, June 2015) to identify $\geq 12$-week, randomized, placebo-controlled trials on DPP-4 inhibitors in $\geq 50$ patients with T2DM and RI. Outcomes of interest included change in glycated hemoglobin (HbA1c), overall safety, and incidence of hypoglycemic events (HEs).

Results: Seven trials of $\leq 52-54$ weeks duration were retrieved, which included one study each on vildagliptin, saxagliptin, and sitagliptin, two on linagliptin, and the remaining two were extension studies of vildagliptin and saxagliptin. Majority of patients were on insulin at baseline (53-86\%), except in the sitagliptin study, where approximately $11 \%$ received insulin during the placebo-controlled phase. After 52 weeks, vildagliptin and saxagliptin reduced HbA1c levels by $0.6-0.7 \%$ (baseline $7.8-8.4 \%$ ) versus placebo in the overall population. HbA1c reductions were similar at weeks 12 and 52. In the 12-week, placebo-controlled phase, sitagliptin and 
linagliptin reduced mean $\mathrm{HbA1c}$ by approximately $0.4 \%$ (baseline $7.7-8.1 \%$ ) versus placebo. Rates of HEs with DPP-4 inhibitors were not significantly different versus placebo in any study. Rates of adverse events (AEs) and changes involving renal function were similar in the active- and placebo-treated groups.

Conclusion: These results suggest that DPP-4 inhibitors have the potential to improve glycemic control in patients with RI without increasing the risk of HEs or overall AEs.

Funding: Novartis Pharma AG.

Keywords: DPP-4 inhibitors; Linagliptin; Saxagliptin; Sitagliptin; Type 2 diabetes mellitus; Vildagliptin

\section{INTRODUCTION}

Renal impairment (RI) is a common complication in patients with type 2 diabetes mellitus (T2DM). Approximately one in every five patients with T2DM has an estimated glomerular filtration rate (eGFR) $<60 \mathrm{~mL} / \mathrm{min} /$ $1.73 \mathrm{~m}^{2}$ [1]. Presence and severity of RI in patients with $\mathrm{T} 2 \mathrm{DM}$ are associated with an increased risk of adverse clinical outcomes, including hospitalization, cardiovascular events, heart failure, end-stage renal disease (ESRD), and premature mortality [2, 3]. Given this elevated risk, there is a strong exigency to intervene in patients with RI. Indeed, studies suggest that a greater reduction in the absolute risks in this population may be achieved with intensive treatment of blood pressure or lipid levels, and fewer patients may need treatment to prevent one event, compared with individuals with normal renal function who required treatment $[4,5]$. However, intensive control of blood glucose levels in patients with T2DM and $\mathrm{RI}$ is challenging and often regarded as problematic, which also compromises glycemic control leading to therapeutic inertia. A robust association exists between glycated hemoglobin (HbA1c) and clinical outcomes in patients with RI [2]; however, in patients with T2DM, presence of RI is associated with an increase in the risk of adverse events (AEs) such as hypoglycemia [6], gastrointestinal symptoms [7], fluid retention [8], and bone fracture [9]. In addition, RI directly or indirectly affects the pharmacokinetics and pharmacodynamics of several glucose-lowering agents and demands dose adjustment while prescribing these agents in patients with RI [10]. Some glucose-lowering agents such as sodium glucose cotransporter-2 (SGLT-2) inhibitors are less effective in patients with RI [9]. Moreover, a high pill burden and the challenges of treatment compliance may also make treatment intensification even more difficult in patients with RI [11]. Dipeptidyl peptidase-4 (DPP-4) inhibitors are widely used in the management of T2DM and have confirmed efficacy and safety, without increasing the risk of hypoglycemia and weight gain in patients with normal renal function [12]. In addition, DPP-4 inhibition has the potential to address several impediments associated with intensification of glycemic control in patients with RI [13]. However, limited data on comparative efficacy and safety of DPP-4 inhibitors are available, especially in patients with RI. Consequently in this paper, we report the findings from a systematic literature review of the efficacy and safety of DPP-4 inhibitors in patients with T2DM and RI.

\section{METHODS}

\section{Search}

We conducted a search of all English language studies on DPP-4 inhibitors in patients with 
T2DM and RI in the databases EMBASE, MEDLINE, and the Cochrane Central Register of Controlled Trials, from database inception to June 3, 2015. Search terms included population (diabetes mellitus, type 2 OR diabet*) AND interventions (vildagliptin, sitagliptin, saxagliptin, linagliptin, alogliptin, gemigliptin, or teneligliptin, their associated molecules or chemical compound/entity names) and randomized controlled trials using the Scottish Intercollegiate Guidelines Network (SIGN)-approved filter. Duplicate publications were eliminated and eligible records were screened independently by two reviewers (from Analysis Group Inc., Boston, MA, USA), first by title and abstract followed by full text. The resulting publications were screened further for studies that were placebo-controlled randomized clinical trials of $\geq 12$-week duration and included $\geq 50$ patients with T2DM and RI who had received a DPP-4 inhibitor, with one or more of the following study outcomes: HbA1c, fasting plasma glucose (FPG), weight, hypoglycemia and lipid profile (complete inclusion and exclusion criteria are shown in Table S1 in the supplementary material).

\section{Outcomes}

The outcomes included changes in HbA1c and FPG at weeks 12 and 52. Overall safety, incidence of hypoglycemic events (HEs) and renal function were also reported.

\section{Additional Analyses}

In the vildagliptin studies $[14,15], \mathrm{HbA1c}$ and FPG outcomes were separately analyzed for patients with moderate (eGFR, $\geq 30$ to $<50 \mathrm{~mL}$ / $\min / 1.73 \mathrm{~m}^{2}$ ) and severe RI (eGFR, $<30 \mathrm{~mL} /$ $\min / 1.73 \mathrm{~m}^{2}$ ) by treatment, but not pooled for overall patients, whereas in sitagliptin [16] and saxagliptin [17, 18] studies, these outcomes were not only separately analyzed for patients with moderate (creatinine clearance $[\mathrm{CrCl}] \geq 30$ to $<50 \mathrm{~mL} / \mathrm{min})$ and severe $\mathrm{RI}(\mathrm{CrCl}<30 \mathrm{~mL} /$ min and not receiving dialysis) but also pooled for overall patients based on treatment. For the present systematic review, an analysis was performed to determine the weighted estimated overall mean HbA1c and FPG, so that the overall data for each vildagliptin study could be compared with other DPP-4 inhibitors.

In trials which did not report numerical values for changes in HbA1c and FPG versus baseline, numerical values were approximated from graphically presented data by pixel analysis. Similarly, if the data on between-treatment differences for changes in HbA1c and FPG were not available in an individual publication; descriptive statistics were used to determine the same. Microsoft Excel (Microsoft Corporation, Redmond, WA, USA) was used for all statistical analyses.

\section{Compliance with Ethics Guidelines}

This article is based on previously conducted studies and does not involve any new studies of human or animal subjects performed by any of the authors.

\section{RESULTS}

\section{Identified Studies}

Overall, we identified 4542 studies during the initial search (Fig. 1), among which 51 studies were shortlisted following deletion of duplicates 
and screening based on titles and abstracts. Of these 51 studies, 7 studies met the inclusion criteria during the screening based on full text: 5 were placebo-controlled trials (one each on vildagliptin [14], sitagliptin [16], and saxagliptin [17], and two on linagliptin $[19,20])$, and the remaining two were 52 -week extension studies of vildagliptin [15] and saxagliptin [18]. The study designs of the studies included in this review are summarized in the supplementary material (Table S2). There were no full-text publications available for gemigliptin, alogliptin, or teneligliptin that met the inclusion criteria, at the time when the search was conducted.

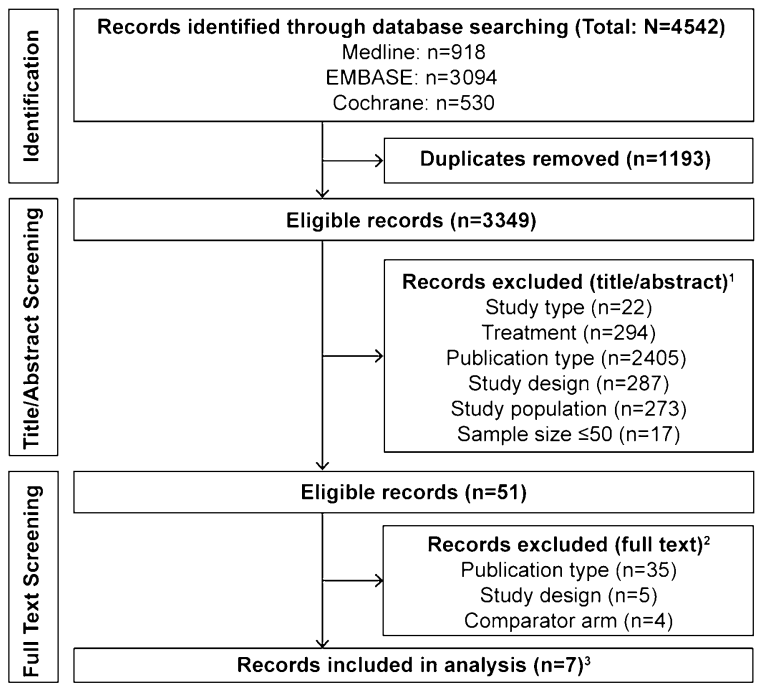

Fig. 1 PRISMA diagram for study selection. 1 The following criteria were applied for screening of title/ abstract but did not lead to any exclusions: outcomes, trial length and comparator arms; 2 the following criteria were applied for screening of full text but did not lead to any exclusions: study type, treatment, outcomes, trial length $\leq 12$ weeks, and sample size $\leq 50 ; 3$ two studies are extensions of other studies included in the analysis after screening of full text $[15,18]$. Relevant studies that only report outcomes for renally impaired patients with type 2 diabetes mellitus in the full text may have been excluded if the title or abstract of the study does not mention this subpopulation

\section{Patient Demographics and Baseline Characteristics}

Demographics and baseline characteristics of patients were broadly similar across treatment groups and within each study, with mean age ranging from 64 to 70 years (Table 1). Over 50\% patients across the study groups were men, except in the saxagliptin study, which had more women (approximately 62\%). Most study participants were White, except the sitagliptin study, which had similar proportions of White, Hispanic/Latino, and Asian participants. The mean body mass index across the groups was approximately $30 \mathrm{~kg} / \mathrm{m}^{2}$, except in the sitagliptin group (approximately $27 \mathrm{~kg} / \mathrm{m}^{2}$ ). At baseline, the mean HbA1c was $<8 \%$ in the sitagliptin and vildagliptin studies (approximately $7.7 \%$ ), while it was $>8 \%$ in the saxagliptin and linagliptin studies (8.1-8.5\%). The mean FPG across the treatment groups ranged between 8.1 and $10.4 \mathrm{mmol} / \mathrm{L}$, with highest levels observed in the saxagliptin group. Patients in the saxagliptin, sitagliptin and vildagliptin studies had a mean T2DM duration of $\geq 13$ years. The disease duration was markedly different in the linagliptin study: most patients (placebo group, 97\%; active treatment group, 95.2\%) had T2DM for more than 5 years.

Apart from the sitagliptin study, most patients in the other studies were on insulin (53-86\%), few received oral antidiabetes drugs (OADs; $14-35 \%$ ), and the remaining received insulin in combination with OADs. However, in the sitagliptin study, most patients (approximately 69\%) received OADs alone and only approximately $11 \%$ received insulin in the placebo-controlled phase of the trial. In the vildagliptin study, almost all patients were on anti-hypertensive agents that block the renin-angiotensin-aldosterone system (RAAS). 


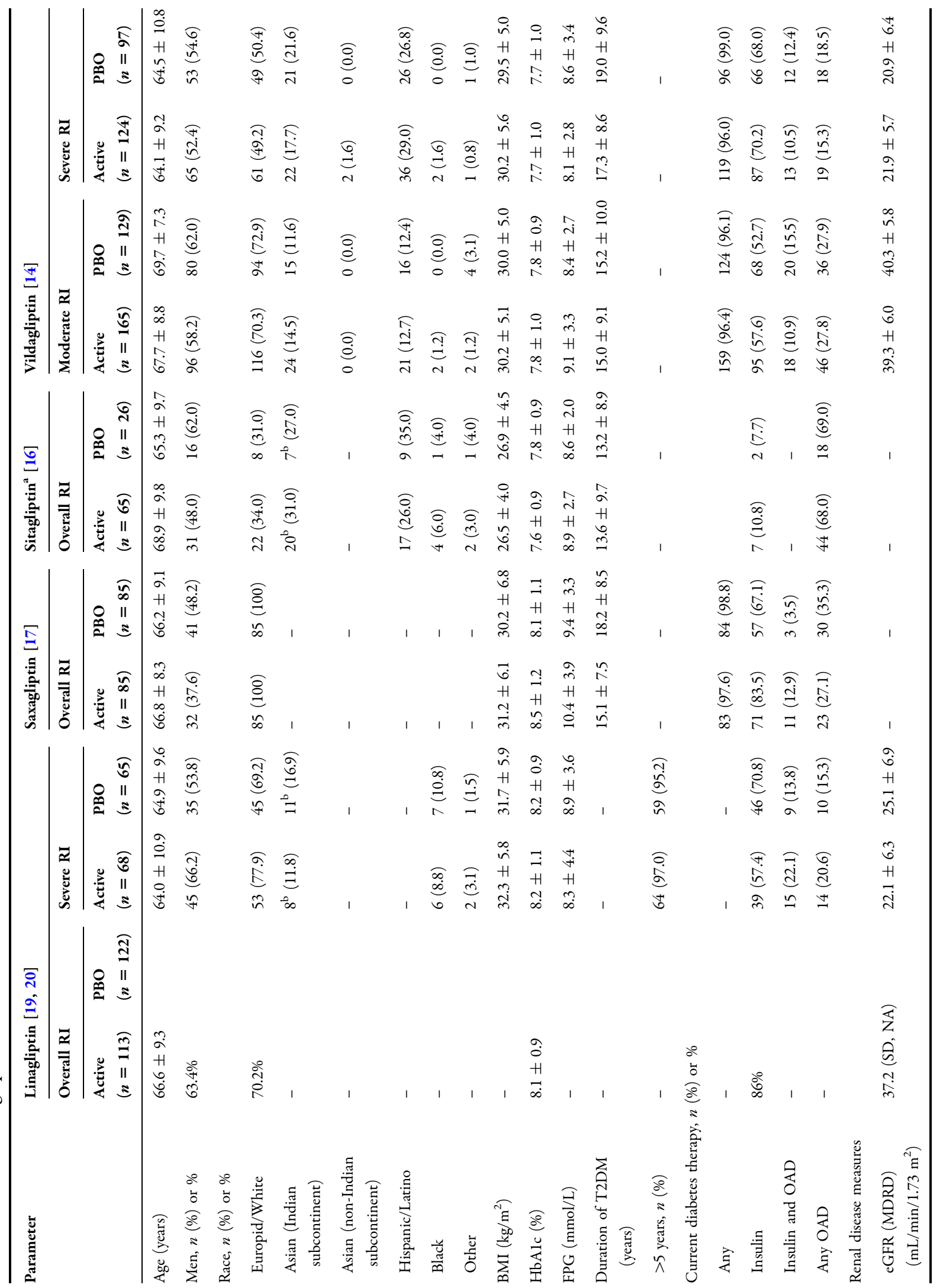




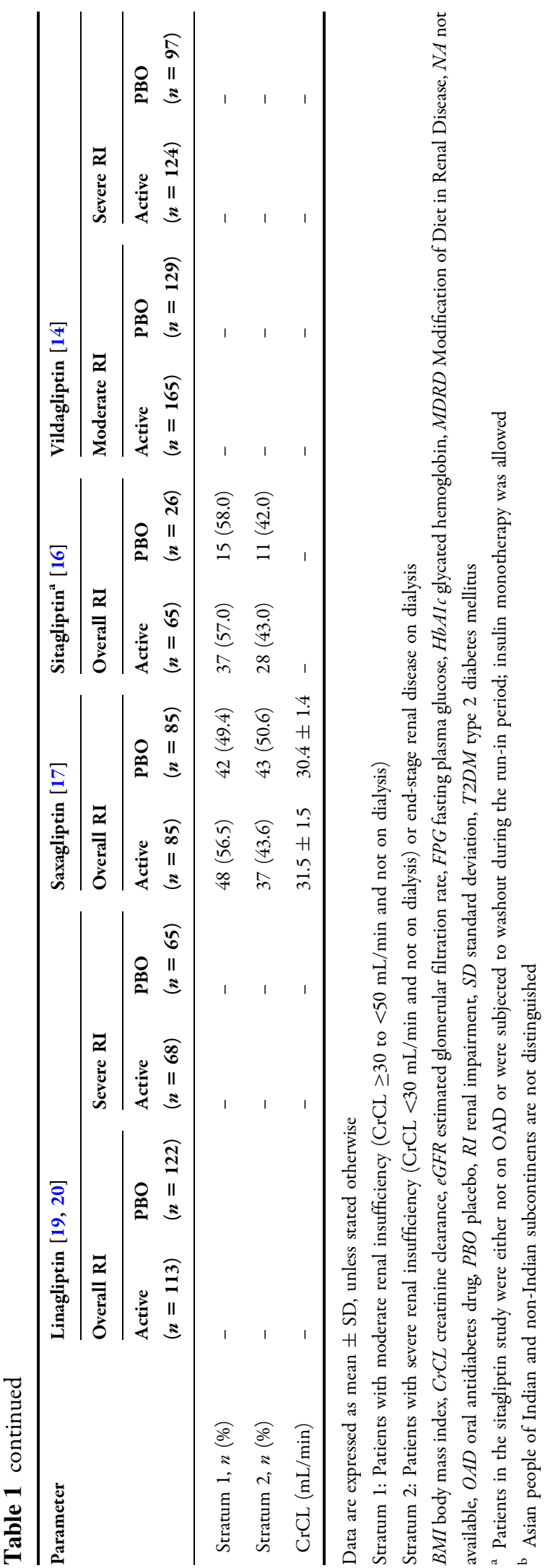

Similarly, in the sitagliptin study, the most frequent concomitant medication was a RAAS inhibitor (>70\%) and in the linagliptin study, $>90 \%$ patients were on anti-hypertensive agent. In the saxagliptin study, use of any concomitant medications other than glucose-lowering agents was not reported.

In the sitagliptin study, open-label rescue therapy (insulin up-titration or initiation of sulfonylurea or insulin) was available throughout the study if pre-specified glycemic control criteria were not met [16]. In the vildagliptin study, rescue medication, as insulin addition or intensification was permitted after 4 weeks if pre-specified glycemic control criteria were not met $[14,15]$. In the saxagliptin study, patients were discontinued from the study if pre-specified glycemic control criteria were not met $[17,18]$. In the linagliptin study, stable doses of background therapy were maintained in the first 12 weeks of the study, unless dose adjustments were necessary due to safety reasons, and in the following 40-week treatment period, background therapy could be adjusted according to glucose parameters; rescue therapy (insulin addition) was allowed based on failure to meet pre-specified glycemic response criteria at any time point during the study [19].

\section{Reduction in HbA1c}

In each long-term study, the HbA1c-lowering effect from a given baseline was similar at weeks 12 and 52 in the overall population (both moderate and severe RI, Fig. 2a), implying sustainable effects in this setting. In the 12-week sitagliptin study, a placebo-adjusted mean $\mathrm{HbA} 1 \mathrm{c}$ reduction of $0.4 \%$ (baseline, $7.6 \%$; $95 \%$ confidence interval [CI], -0.7 to -0.1 ) was observed in the overall population [16]. The 
Fig. 2 Mean HbAlc levels in patients with T2DM and RI at weeks 12 and 52. a Overall RI; b moderate RI; and c severe RI. Data are presented as mean HbAlc levels at weeks 12 and 52 from the baseline. Cap indicates starting of baseline values, downward arrow indicates magnitude of reduction from baseline and upward arrow indicates magnitude of increase from baseline. ${ }^{\wedge}$ Included patients with moderate RI and severe RI. ${ }^{*}$ Included patients with moderate RI, severe RI, and ESRD. Overall data for vildagliptin study was estimated by weighted average mean of patients with moderate and severe RI. Numerical values for mean $\mathrm{HbAlc}$ levels at week 12 for vildagliptin studies were approximated from graphically presented data by pixel analysis. ESRD end-stage renal disease, $H b A I c$ glycated hemoglobin, LINA linagliptin, $P B O$ placebo, $R I$ renal impairment, SAXA saxagliptin, SITA sitagliptin, $T 2 D M$ type 2 diabetes mellitus, VILDA vildagliptin

placebo-adjusted mean reduction in $\mathrm{HbA} 1 \mathrm{c}$ with vildagliptin was $0.6 \%$ (baseline, $7.8 \%$ ) at week 12 , which was sustained until 52 weeks $[14,15]$. The placebo-adjusted mean reduction in HbA1c was significantly high in the saxagliptin group at week $12(0.42 \%$; baseline, $8.5 \% ; 95 \% \mathrm{CI},-0.71$ to $-0.12 ; P=0.007)$ and week 52 (0.73\%; baseline, 8.4\%; 95\% CI, -1.11 to $-0.34 ; P<0.001)[17,18]$. After 12 weeks, the placebo-adjusted mean reduction in $\mathrm{HbA1c}$ with linagliptin was $0.42 \%$ (95\% CI, -0.60 to $-0.24 ; P<0.0001$ ) [20].

In patients with moderate RI, the placebo-adjusted mean reduction in $\mathrm{HbA1c}$ in vildagliptin-treated patients at week 12 was $0.63 \%$ and at week 52 was $0.4 \%$, which was statistically significant $(P=0.005 ; \quad$ Fig. $2 b)$ $[14,15]$. Similarly, saxagliptin-treated patients showed numerically higher placebo-adjusted mean reductions in $\mathrm{HbA1c}$ at weeks 12 $(0.59 \%)$ and $52(1.13 \%)$ [17, 18]. HbA1c data for sitagliptin- and linagliptin-treated patients with moderate RI are not available in their individual publications $[16,19,20]$.

In patients with severe RI treated with vildagliptin, the placebo-adjusted mean

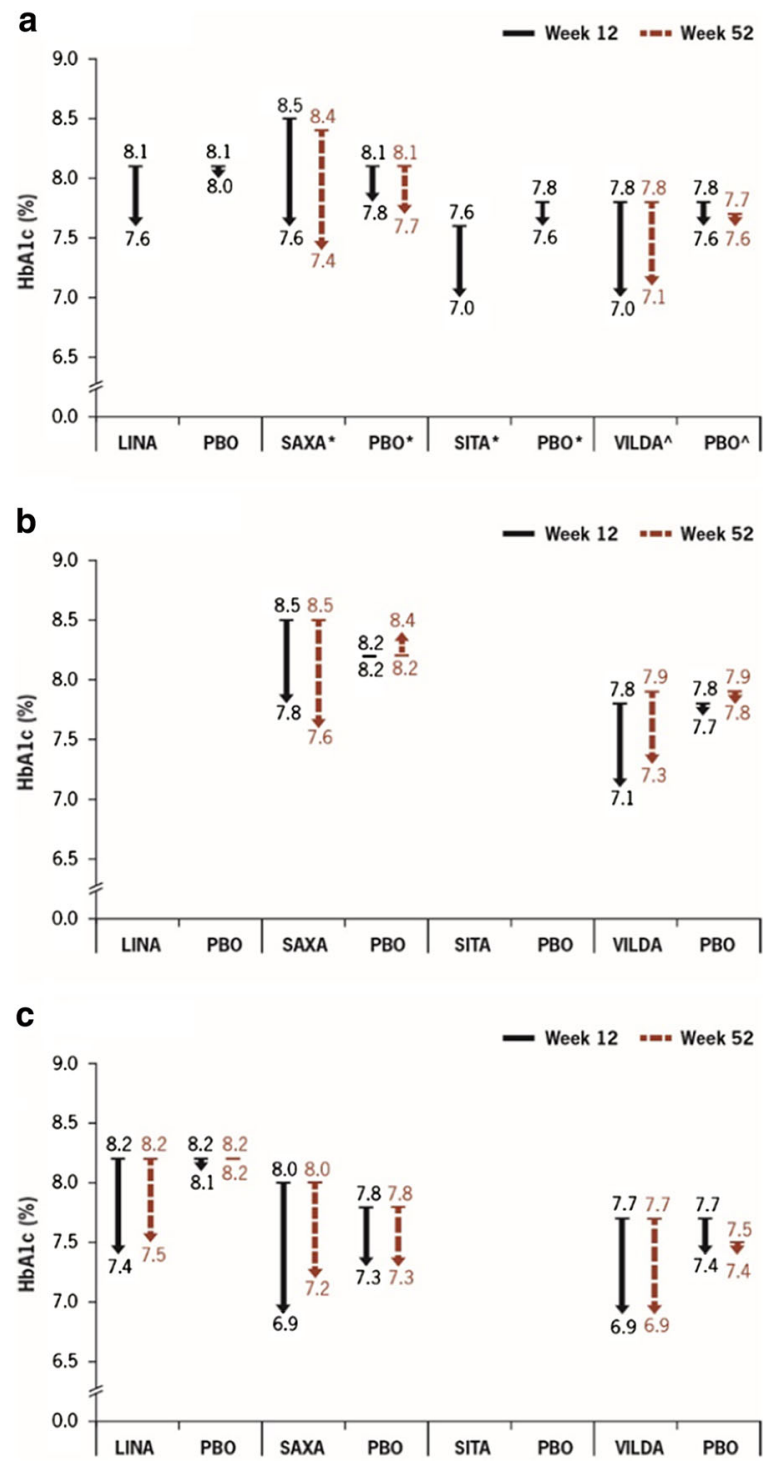

reduction in $\mathrm{HbA} 1 \mathrm{c}$ of $0.5 \%$ at week 12 and $0.7 \%(P<0.0001)$ at week 52 was significant (Fig. 2c) [14, 15]. Saxagliptin-treated patients showed a numerically greater reduction in HbA1c versus placebo of $0.45 \%$ and $0.32 \%$ at weeks 12 and 52, respectively [17, 18]. The between-treatment difference for linagliptin versus placebo was statistically significant at weeks 12 ( $-0.60 \%$; 95\% CI, -0.89 to -0.31 ; $P<0.0001)$ and $52(-0.72 \% ; 95 \% \mathrm{CI},-1.03$ to $-0.41 ; P<0.0001)[19]$. 
Fig. 3 Mean FPG levels in patients with T2DM and RI at weeks 12 and 52. a Overall RI; b moderate RI; and c severe RI. Data are presented as mean FPG levels at weeks 12 and 52 from the baseline. Cap indicates starting of baseline values; downward arrow indicates magnitude of reduction from baseline; upward arrow indicates magnitude of increase from baseline. ${ }^{\wedge}$ Included patients with moderate $\mathrm{RI}$ and severe RI. * Included patients with moderate RI, severe RI, and ESRD. Overall data for vildagliptin study was estimated by weighted average mean of patients with moderate and severe RI. Numerical values for mean FPG levels at week 12 for vildagliptin studies were approximated from graphically presented data by pixel analysis. ESRD end-stage renal disease, FPG fasting plasma glucose, $L I N A$ linagliptin, $P B O$ placebo, $R I$ renal impairment, $S A X A$ saxagliptin, SITA sitagliptin, T2DM type 2 diabetes mellitus, $V I L D A$ vildagliptin

Data for patients with ESRD were available only for the saxagliptin study. At week 12, the between-group difference in HbA1c was $0.03 \%$ ( $P$ value non-significant), and after 52 weeks, this difference was $-0.14 \%$ (data not shown) $[17,18]$. There was a modest $(0.1-0.2 \%)$ but significantly greater reduction in $\mathrm{HbA1c}$ in patients with severe RI (eGFR, $<30 \mathrm{~mL} / \mathrm{min} /$ $1.73 \mathrm{~m}^{2}$ ) than in patients with moderate RI (eGFR, 30-60 mL/min/1.73 $\mathrm{m}^{2} ; P<0.05$ ).

\section{Reduction in FPG}

The mean reductions in FPG from baseline at weeks 12 and 52 in the overall population of each study are depicted in Fig. 3a; further stratifications by moderate RI (Fig. 3b) and severe RI (Fig. 3c) subgroups are also shown. In the 12-week sitagliptin study, the difference in FPG between sitagliptin- and placebo-treated patients was $-1.3 \mathrm{mmol} / \mathrm{L}$ (95\% CI, -2.2 to $-0.3)$ [16].

\section{Safety and Tolerability}

Overall, the incidences of AEs were similar between the active treatment and placebo

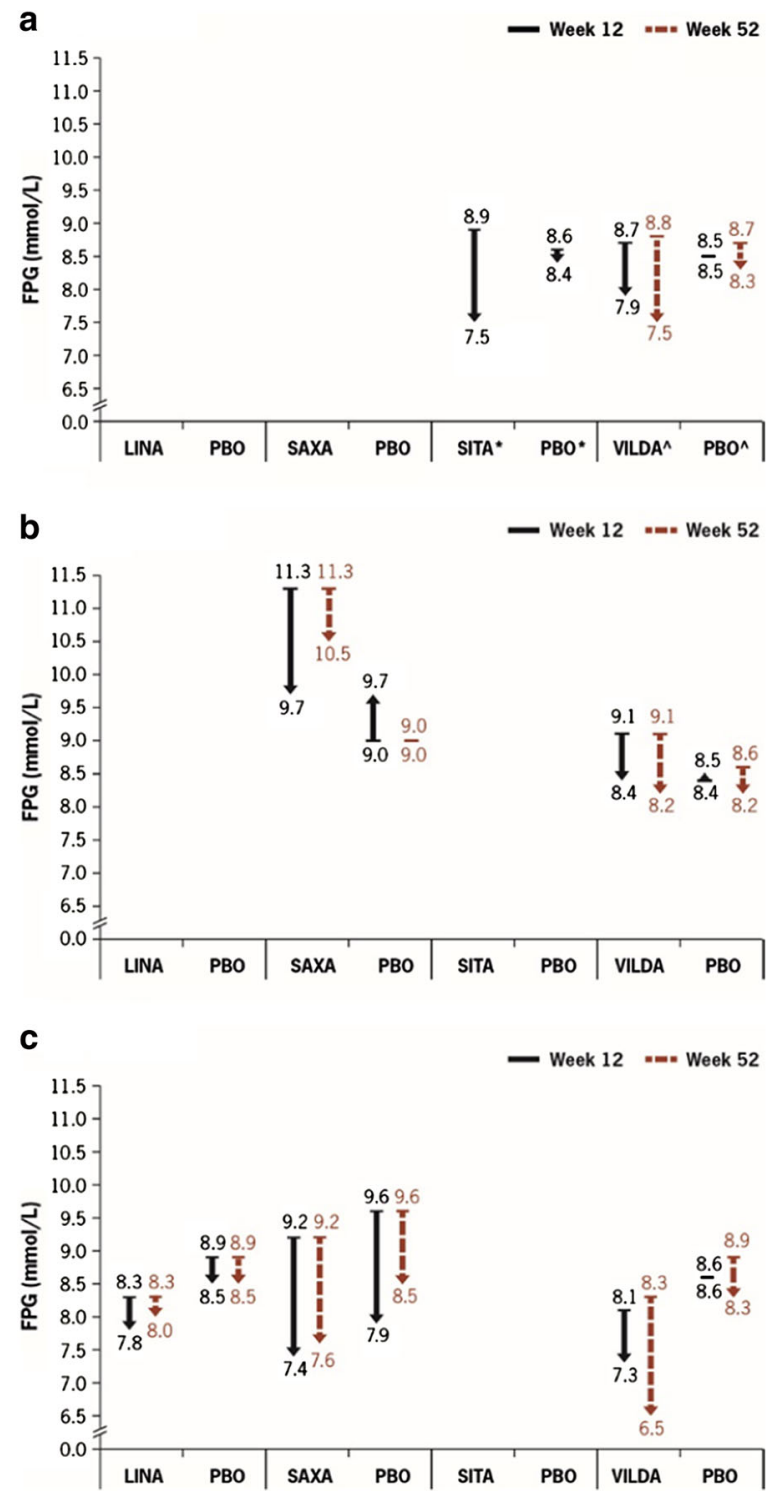

groups across all studies (Table 2). The number of patients reporting any $\mathrm{AE}$, drug-related AEs, serious AEs, and AEs leading to treatment discontinuation in the treatment groups were broadly similar to that of their corresponding placebo groups, and AEs were mild to moderate in severity [14-20].

In every study, the most frequently reported AE was hypoglycemia (Table 3). For sitagliptin, during the 12-week, placebo-only controlled phase of the trial, the incidence of hypoglycemia was similar between both 


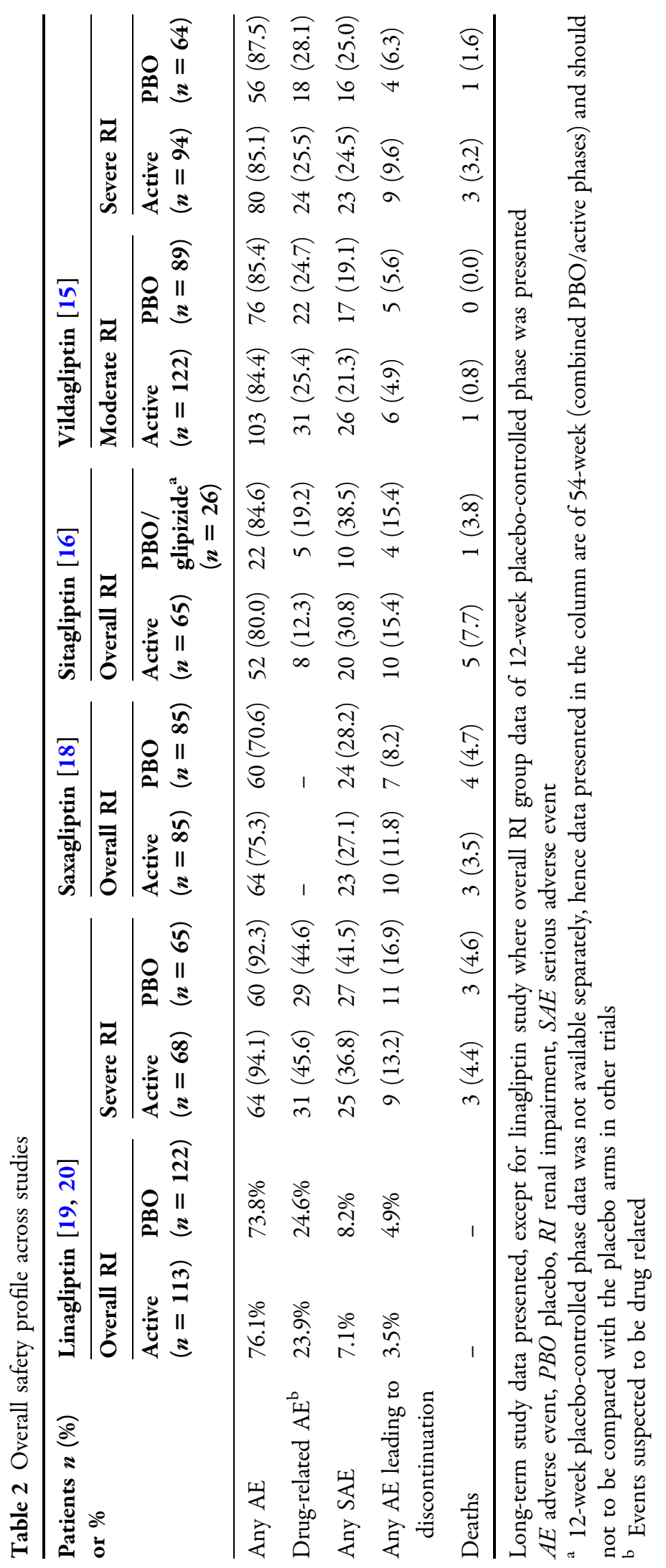




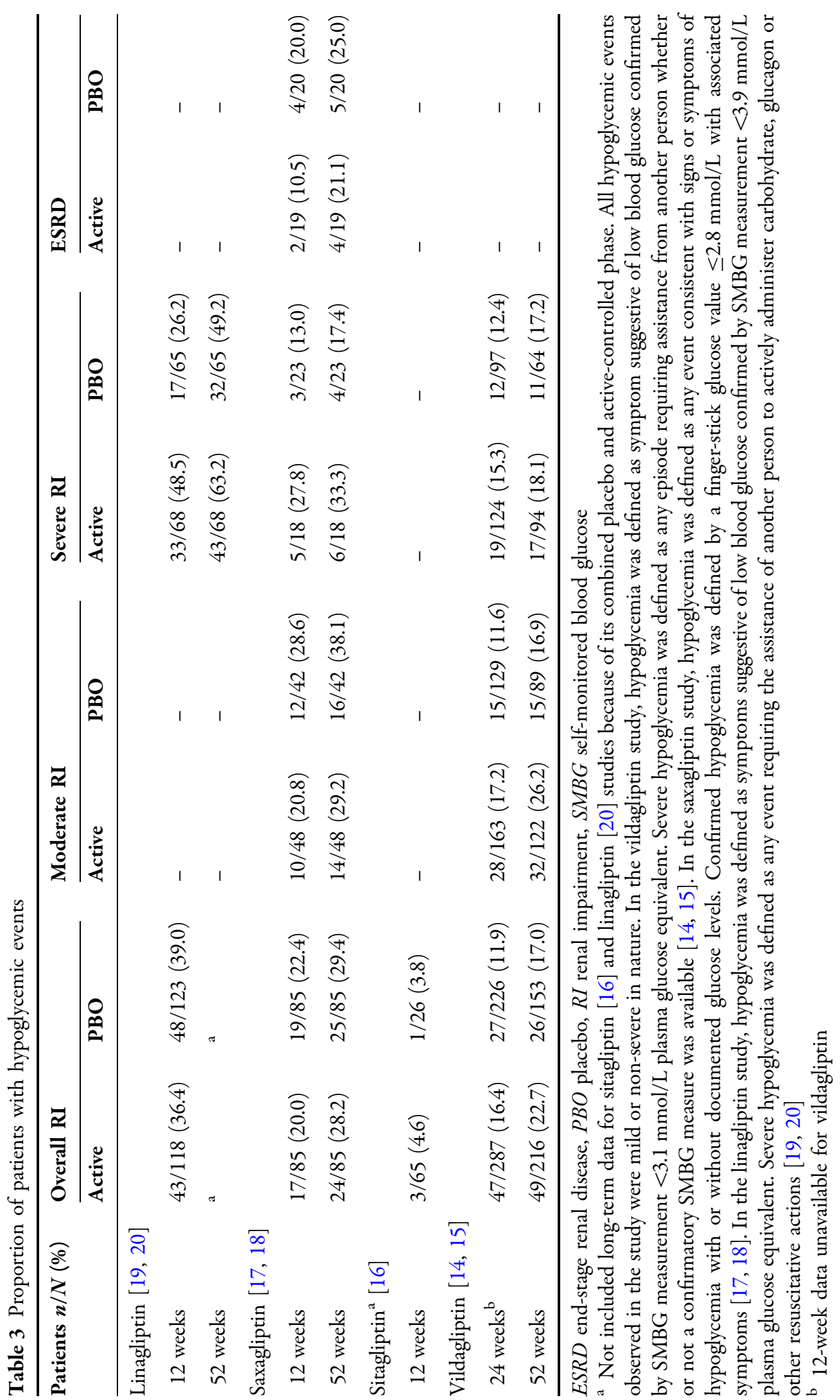


groups (sitagliptin, 4.6\% vs. placebo, 3.8\%) [16]. In the vildagliptin study, the incidences of hypoglycemia in the overall population at weeks 24 and 52 were comparable with placebo $[14,15]$. In patients with moderate RI, the incidence of HEs was numerically higher in patients who received active treatment versus those who received placebo at weeks 24 and 52 . The incidence of severe hypoglycemia was similar between the treatments (vildagliptin, $1.2 \%$ vs. placebo, $1.6 \%$ ) at week 24 [14]. In the severe RI group, the incidences of hypoglycemia were similar with vildagliptin and placebo at both weeks 24 and 52 [14, 15]. However, the incidence of severe hypoglycemia was lower with vildagliptin than placebo at week 52 in patients with both moderate and severe RI [15]. In the saxagliptin study, the incidences of hypoglycemia were similar in both active and placebo groups at weeks 12 and 52 in the overall population and in patients with moderate RI and ESRD $[17,18]$. However, in patients with severe RI, the incidence of hypoglycemia in the saxagliptin-treated patients was almost double that in the placebo-treated patients at weeks 12 (27.8\% vs. $13.0 \%)$ and 52 (33.3\% vs. $17.4 \%)$ $[17,18]$. In the 12-week placebo-controlled phase, the incidence of hypoglycemia was comparable between linagliptin and placebo in the overall population [20]. In another study, the incidence of hypoglycemia was higher in linagliptin-treated patients with severe RI than in placebo-treated patients at weeks 12 and 52; this was attributed to the higher incidence of asymptomatic hypoglycemia with linagliptin [19]. However, the incidence of severe hypoglycemia was low and similar between both groups: $4.4 \%$ with linagliptin and $4.6 \%$ with placebo [19].

\section{Renal Findings}

In the vildagliptin study, there was a slight but similar decline in eGFR over 52 weeks in both vildagliptin and placebo groups (moderate RI, -1.62 vs. $-1.80 \mathrm{~mL} / \mathrm{min}$; severe RI, -1.98 vs. $-2.44 \mathrm{~mL} / \mathrm{min}$, respectively). The mean serum potassium levels were more or less unchanged over the course of the year. The incidence of hyperkalemia was comparable between vildagliptin- and placebo-treated patients in both moderate $(3.3 \%$ vs. $3.4 \%)$ and severe RI (10.6\% vs. 6.3\%) groups. This incidence was slightly higher in severe RI group than in moderate RI group [15]. In the saxagliptin study, patients with moderate or severe RI reported a slight decline in eGFR over 52 weeks (details not provided) [18]. The decline in eGFR in the linagliptin study was not clinically meaningful in patients with severe RI [19] and remained stable throughout the study in overall patients with RI [20]; the incidence of hyperkalemia was broadly similar in the linagliptin and placebo groups (30.9\% vs. $24.6 \%$ ) and not judged to be related to the study drug for the linagliptin group [19]. Neither eGFR nor hyperkalemia findings were reported for sitagliptin [16].

\section{DISCUSSION}

DPP-4 inhibitor therapies have substantially contributed to the advancement in the management of T2DM. However, their greatest clinical impact may be found in patients in whom conventional therapy has considerable limitations leading to therapeutic inertia and/or compromises in glycemic control. A prime example has been the use of DPP-4 inhibitors 
in patients with T2DM and RI. In this systematic literature review, we showed that DPP-4 inhibitors are both efficacious and well tolerated in patients with T2DM and RI when used at appropriate doses or dosing frequencies based on the severity of RI. These results further support the use of DPP-4 inhibitors in the particularly challenging setting of RI.

In all the aforementioned studies, DPP-4 inhibitors in patients with RI reduced HbA1c levels by $0.4-0.7 \%$, wherein the mean baseline HbA1c ranged from $7.7 \%$ to $8.4 \%$, compared with placebo at different time points [14-20]. This is comparable to the reductions observed in placebo-controlled trials in patients with normal renal function, particularly when DPP-4 inhibitors were added to pre-existing [21] and often long-term insulin therapy (the most common background therapy in studies included in this analysis). The findings reported in the present review were in line with a previously reported systematic review and meta-analysis of 10 pooled studies conducted in patients with moderate and severe RI [22].

Notably, a modest $(0.1-0.2 \%)$ but greater additional reduction in $\mathrm{HbA1c}$ was observed in patients with severe RI (eGFR, $<30 \mathrm{~mL} / \mathrm{min} /$ $1.73 \mathrm{~m}^{2}$ ) than in those with moderate RI (eGFR, $\left.30-60 \mathrm{~mL} / \mathrm{min} / 1.73 \mathrm{~m}^{2} ; P<0.05\right)$. It is possible to speculate that patients with severe RI may show a greater increase in glucagon-like peptide-1 (GLP-1) as its half-life is further prolonged by reduced renal clearance [23]. In addition, the reduced half-life of erythrocytes in patients with severe RI may have underestimated glycemic control in such patients than in those with better renal function [24]. As the effects of DPP-4 inhibition (and incretin effect) on glycemia are proportionally greater in individuals with higher baseline glucose levels [25], a greater reduction in HbA1c may be anticipated in patients with severe RI who are likely to have had higher glucose levels despite having comparable baseline HbA1c levels to those with moderate RI.

Hypoglycemia, a frequent complication of diabetes management, affects approximately $25 \%$ of patients with T2DM at least once a year [26]. It is also often an important barrier to optimized glycemic control [27]. RI is associated with an increased incidence and severity of HEs [28]. Indeed, in all studies included in the present analysis, $1-4 \%$ of patients with RI experienced a severe HE (requiring assistance). This proportion is 5- to 10-times higher than that reported in patients with normal renal function [29], even without adjusting for the appropriate dose of insulin or insulin secretagogues in this setting. However, the addition of DPP-4 inhibitors in this setting achieved improved glycemic control without any significant increase in HEs in any individual study compared with placebo. Moreover, it is likely that in clinical settings, pre-emptive down-titration of insulin/sulfonylurea dosage following improvement in glycemic control induced by DPP-4 inhibition should result in even lower rates of hypoglycemia in patients receiving DPP-4 inhibitors. However, in blinded placebo-controlled trials, any down-titration is generally limited and would not occur until the patient experiences a HE or records lower glucose levels [14-20].

Moreover, T2DM management in patients with RI is associated with higher health care costs and reduced health-related quality of life $[1,30]$, which is partly mediated by the presence and severity of co-morbid diseases and multi-factorial interventions necessitated by their presence [30]. In addition, the frequency and severity of hypoglycemia as well as the risk of hypoglycemia are critical determinants of overall health status and mental and physical 
health [31]. Although no economic or quality of life indices were assessed in the studies included in this systematic literature review, a previous study has shown that DPP-4 inhibitors may improve quality of life indices in patients with T2DM and normal renal function [32], whereas the introduction or escalation of insulin therapy might reduce them. Health-economic and quality of life studies, specifically in patients with T2DM and RI receiving DPP-4 inhibitors, are certainly warranted for various reasons; different dosing frequencies might have cost implications, and it is well documented that patients with chronic kidney disease not only have generally increased costs but also have a reduced quality of life.

In patients with established RI, avoiding or slowing a further decline in renal function is a priority. In the ADVANCE trial (ClinicalTrials.gov identifier: NCT00145925), an intensified glycemic control significantly reduced the number of individuals requiring renal replacement therapy [33]. Such data support the renoprotective utility of improved glycemic control in patients at the risk of ESRD (i.e., in patients with established RI), provided it can be safely achieved. Unfortunately, as in the ADVANCE study (using the sulfonylurea, gliclazide), the risk of severe hypoglycemia is often increased after intensification in patients with RI [34]; hence, the overall balance of risks and benefits remain unclear. However, the efficacy and safety of glycemic control with DPP-4 inhibitors in patients with RI reported in this analysis should change this balance.

Certain studies have suggested that DPP-4 inhibitors may have renal benefits beyond glucose lowering [35] and have also reported modest reductions in albuminuria [36, 37]. However, it is unclear whether this is a valid marker of renal protection achieved by these agents. In the SAVOR TIMI-53 study
(ClinicalTrials.gov identifier: NCT01107886), despite reduction in albuminuria, there was no difference in ESRD or progressive renal decline between patients receiving saxagliptin and those receiving placebo [37]. At present, it can be concluded that there is no evidence of any adverse effect on renal function following the use of DPP-4 inhibitors (i.e., renal safety) in patients with T2DM and RI [15, 19]. Longer studies with a focus on renal safety remain to be completed to establish any renoprotective effects in addition to glycemic control. However, fundamentally, for the use of DPP-4 inhibitors in patients with RI, the ability to safely lower glucose levels is the primary attribute and indication.

\section{CONCLUSIONS}

In summary, for patients with T2DM and RI, diabetes management is complex, multi-dimensional and potentially expensive. Our systematic review suggests that DPP-4 inhibitors have the potential to improve and simplify glycemic control in this setting without exposure to hypoglycemia or other important AEs. The renoprotective potential of DPP-4 inhibitors remains unproven, but is a subject of ongoing investigations in clinical trials. Since improved glycemic control has been shown to reduce the risk of ESRD, safe and optimum control in patients with RI would also have additional renal benefits. Given the high financial burden and reduced quality of life of patients with T2DM and RI, further economic and quality of life analyses are warranted.

\section{ACKNOWLEDGMENTS}

This research was supported by Novartis Pharma AG, Basel, Switzerland. The article processing 
charges for this publication were funded by Novartis Pharma AG. All authors participated in the study design, data review and data interpretation. All named authors meet the International Committee of Medical Journal Editors (ICMJE) criteria for authorship for this manuscript, take responsibility for the integrity of the work as a whole, and have given final approval to the version to be published. All authors were involved in preparing and revising manuscript and have reviewed the final draft. All authors are responsible for intellectual content and data accuracy. The authors acknowledge the patients, investigators and staff at participating sites for all the studies. We thank Anuja Shah (Novartis Healthcare Pvt. Ltd., Hyderabad, India) and Cathy McDonnell (Novartis Ireland Ltd., Dublin, Ireland) for manuscript preparation, incorporating and collating the comments from the authors and editorial assistance.

Disclosures. Merlin Thomas has received honoraria for educational symposia and expert panels provided on behalf of AstraZeneca, Abbott, Reata, AbbVie, Sanofi Aventis, Bristol-Myers Squibb, Boehringer Ingelheim, Eli Lilly, Merck and Co., Servier, Janssen-Cilag and Amgen. Päivi Maria Paldánius, is employed by and own shares in Novartis. Rajeev Ayyagari is an employee of Analysis Group, Inc., which received funding from Novartis for conducting this study. Siew Hwa Ong is an employee of Vifor Pharma Ltd., and was employed by Novartis at the time of manuscript development. Per-Henrik Groop has received lecture honoraria/advisory board fees from AbbVie, Boehringer Ingelheim, Cebix, Eli Lilly, Genzyme, Janssen, Medscape, Merck and Co., Novartis, Novo Nordisk, and Medscape and research grants from Eli Lilly and Roche.
Compliance with Ethics Guidelines. This article is based on previously conducted studies and does not involve any new studies of human or animal subjects performed by any of the authors.

Open Access. This article is distributed under the terms of the Creative Commons Attribution-NonCommercial 4.0 International License (http://creativecommons.org/licenses/ by-nc/4.0/), which permits any noncommercial use, distribution, and reproduction in any medium, provided you give appropriate credit to the original author(s) and the source, provide a link to the Creative Commons license, and indicate if changes were made.

\section{REFERENCES}

1. Thomas MC, Brownlee M, Sustak K, et al. Diabetic kidney disease. Nat Rev Dis Primers. 2015;. doi:10. 1038/nrdp.2015.18.

2. Shurraw S, Hemmelgarn B, Lin M, et al. Association between glycemic control and adverse outcomes in people with diabetes mellitus and chronic kidney disease: a population-based cohort study. Arch Intern Med. 2011;171(21):1920-7.

3. Tonelli M, Muntner P, Lloyd A, et al. Risk of coronary events in people with chronic kidney disease compared with those with diabetes: a population-level cohort study. Lancet. 2012;380(9844):807-14.

4. Perkovic V, Ninomiya $\mathrm{T}$, Arima $\mathrm{H}$, et al. Chronic kidney disease, cardiovascular events, and the effects of perindopril-based blood pressure lowering: data from the PROGRESS study. J Am Soc Nephrol. 2007;18(10):2766-72.

5. Shepherd J, Kastelein JJ, Bittner V, et al. Effect of intensive lipid lowering with atorvastatin on renal function in patients with coronary heart disease: the treating to new targets (TNT) study. Clin J Am Soc Nephrol. 2007;2(6):1131-9.

6. Weir MA, Gomes T, Mamdani M, et al. Impaired renal function modifies the risk of severe hypoglycaemia among users of insulin but not 
glyburide: a population-based nested case-control study. Nephrol Dial Transpl. 2011;26(6):1888-94.

7. Linnebjerg H, Kothare PA, Park S, et al. Effect of renal impairment on the pharmacokinetics of exenatide. Br J Clin Pharmacol. 2007;64(3):317-27.

8. Kohan DE, Lambers Heerspink HJ, Coll B, et al. Predictors of atrasentan-associated fluid retention and change in albuminuria in patients with diabetic nephropathy. Clin J Am Soc Nephrol. 2015;10(9):1568-74.

9. Kohan DE, Fioretto P, Tang W, List JF. Long-term study of patients with type 2 diabetes and moderate renal impairment shows that dapagliflozin reduces weight and blood pressure but does not improve glycemic control. Kidney Int. 2014;85(4):962-71.

10. Scheen AJ. Pharmacokinetic considerations for the treatment of diabetes in patients with chronic kidney disease. Expert Opin Drug Metab Toxicol. 2013;9(5):529-50.

11. Kennedy M, Roberts A. Complex type 2 diabetes mellitus-management challenges and pitfalls. Aust Fam Physician. 2013;42(4):207-10.

12. Davidson JA. The placement of DPP-4 inhibitors in clinical practice recommendations for the treatment of type 2 diabetes. Endocr Pract. 2013;19(6):1050-61.

13. Game F. Novel hypoglycaemic agents: considerations in patients with chronic kidney disease. Nephron Clin Pract. 2014;126(1):14-8.

14. Lukashevich V, Schweizer A, Shao Q, et al. Safety and efficacy of vildagliptin versus placebo in patients with type 2 diabetes and moderate or severe renal impairment: a prospective 24-week randomized placebo-controlled trial. Diabetes Obes Metab. 2011;13(10):947-54.

15. Kothny W, Shao Q, Groop PH, Lukashevich V. One-year safety, tolerability and efficacy of vildagliptin in patients with type 2 diabetes and moderate or severe renal impairment. Diabetes Obes Metab. 2012;14(11):1032-9.

16. Chan JC, Scott R, Arjona Ferreira JC, et al. Safety and efficacy of sitagliptin in patients with type 2 diabetes and chronic renal insufficiency. Diabetes Obes Metab. 2008;10(7):545-55.

17. Nowicki M, Rychlik I, Haller H, et al. Saxagliptin improves glycaemic control and is well tolerated in patients with type 2 diabetes mellitus and renal impairment. Diabetes Obes Metab. 2011;13(6):523-32.
18. Nowicki M, Rychlik I, Haller H, et al. Long-term treatment with the dipeptidyl peptidase- 4 inhibitor saxagliptin in patients with type 2 diabetes mellitus and renal impairment: a randomised controlled 52-week efficacy and safety study. Int J Clin Pract. 2011;65(12):1230-9.

19. McGill JB, Sloan L, Newman J, et al. Long-term efficacy and safety of linagliptin in patients with type 2 diabetes and severe renal impairment: a 1-year, randomized, double-blind, placebo-controlled study. Diabetes Care. 2013;36(2):237-44.

20. Laakso M, Rosenstock J, Groop PH, et al. Treatment with the dipeptidyl peptidase-4 inhibitor linagliptin or placebo followed by glimepiride in patients with type 2 diabetes with moderate to severe renal impairment: a 52-week, randomized, double-blind clinical trial. Diabetes Care. 2015;38(2):e15-7.

21. Scheen AJ. A review of gliptins for 2014. Expert Opin Pharmacother. 2015;16(1):43-62.

22. Cheng D, Fei Y, Liu Y, et al. Efficacy and safety of dipeptidyl peptidase- 4 inhibitors in type 2 diabetes mellitus patients with moderate to severe renal impairment: a systematic review and meta-analysis. PLoS One. 2014;9(10):e111543.

23. Idorn T, Knop FK, Jorgensen MB, et al. Elimination and degradation of glucagon-like peptide- 1 and glucose-dependent insulinotropic polypeptide in patients with end-stage renal disease. J Clin Endocrinol Metab. 2014;99(7):2457-66.

24. Chujo K, Shima K, Tada H, Oohashi T, Minakuchi J, Kawashima S. Indicators for blood glucose control in diabetics with end-stage chronic renal disease: $\mathrm{GHb}$ vs. glycated albumin (GA). J Med Invest. 2006;53(3-4):223-8.

25. Bloomgarden ZT, Dodis R, Viscoli CM, Holmboe ES, Inzucchi SE. Lower baseline glycemia reduces apparent oral agent glucose-lowering efficacy: a meta-regression analysis. Diabetes Care. 2006;29(9):2137-9.

26. Cryer PE. Hypoglycemia: still the limiting factor in the glycemic management of diabetes. Endocr Pract. 2008;14(6):750-6.

27. Choudhary P, Amiel SA. Hypoglycaemia: current management and controversies. Postgrad Med J. 1026;2011(87):298-306.

28. Alsahli M, Gerich JE. Hypoglycemia, chronic kidney disease, and diabetes mellitus. Mayo Clin Proc. 2014;89(11):1564-71. 
29. Simon D, Detournay B, Eschwege E, et al. Use of vildagliptin in management of type 2 diabetes: effectiveness, treatment persistence and safety from the 2-year real-life VILDA study. Diabetes Ther. 2014;5(1):207-24.

30. Vupputuri S, Kimes TM, Calloway MO, et al. The economic burden of progressive chronic kidney disease among patients with type 2 diabetes. J Diabetes Complicat. 2014;28(1):10-6.

31. Shi L, Shao H, Zhao Y, Thomas NA. Is hypoglycemia fear independently associated with health-related quality of life? Health Qual Life Outcomes. 2014;12:167.

32. Sakamoto Y, Oyama J, Ikeda H, et al. Effects of sitagliptin beyond glycemic control: focus on quality of life. Cardiovasc Diabetol. 2013;12:35.

33. Perkovic V, Heerspink HL, Chalmers J, et al. Intensive glucose control improves kidney outcomes in patients with type 2 diabetes. Kidney Int. 2013;83(3):517-23.
34. Zoungas S, Patel A, Chalmers J, et al. Severe hypoglycemia and risks of vascular events and death. N Engl J Med. 2010;363(15):1410-8.

35. Cooper ME, Perkovic V, McGill JB, et al. Kidney disease end points in a pooled analysis of individual patient-level data from a large clinical trials program of the dipeptidyl peptidase 4 inhibitor linagliptin in type 2 diabetes. Am J Kidney Dis. 2015;66(3):441-9.

36. Groop PH, Cooper ME, Perkovic V, et al. Dipeptidyl peptidase-4 inhibition with linagliptin and effects on hyperglycaemia and albuminuria in patients with type 2 diabetes and renal dysfunction: rationale and design of the MARLINA-T2D trial. Diab Vasc Dis Res. 2015;12(6):455-62.

37. Scirica BM, Bhatt DL, Braunwald E, et al. Saxagliptin and cardiovascular outcomes in patients with type 2 diabetes mellitus. $\mathrm{N}$ Engl J Med. 2013;369(14):1317-26. 